\title{
A quasi-experimental study to test a prevention bundle for catheter-associated urinary tract infections
}

\author{
Alyson W. Blanck, Moreen Donahue, Laurie Brentlinger, Kristy Dixon Stinger, Carol Polito \\ Danbury Hospital, Danbury, CT, USA
}

Correspondence: Alyson W. Blanck. Address: Danbury Hospital, 24 Hospital Ave, Danbury, CT, 06810, USA. E-mail: alyson.blanck@wchn.org

Received: February 15, 2014

Accepted: March 21, 2014

Online Published: March 27, 2014

DOI : $10.5430 /$ jha.v3n4p101

URL: http://dx.doi.org/10.5430/jha.v3n4p101

\begin{abstract}
The purpose of this quasi-experimental study was to test the use of a bundled approach of catheter care practices for a 3-month period to reduce the occurrence of catheter-associated urinary tract infections (CAUTIs) in adult critical care patients who had indwelling urinary catheters. Catheter care is completed primarily by nursing staff, however minimal studies exist in the literature that support specific nursing care to reduce the occurrence of CAUTIs. Catheter care practices were investigated and implemented in a bundled approach to determine the impact on reducing CAUTIs in a critical care unit. Though the results were not statistically significant, they were clinically significant with a $50 \%$ reduction in CAUTI incidence.
\end{abstract}

\section{Key words}

Catheter-associated urinary tract infection, Prevention, Urinary catheter care, Bundled catheter care

\section{I ntroduction}

In the United States, urinary tract infections (UTIs) are the most common healthcare-associated infections, with an estimated annual rate of 560,000 per year; and of this, up to 387,550 are preventable catheter-associated urinary tract infections (CAUTIs) ${ }^{[1]}$. CAUTIs account for $23 \%$ of the healthcare-associated infections in adult critical care units, and they can lead to complications such as cystitis, pyelonephritis, prostatitis, bacteremia, sepsis, and increased risk of death ${ }^{[2-4]}$. Additionally, acute care hospitals must report monthly critical care CAUTI incidence rates as part of the Centers for Medicare and Medicaid Services (CMS) hospital infection reporting requirements. National rates for critical care have reached as high as 8.1 infections per 1000 catheter days ${ }^{[5]}$.

At a 370-bed regional medical center in the Northeast, the annual CAUTI rate in the adult critical care unit (CCU) has been as high as 5.9, which is higher than the NHSN pooled mean of 2.2 for major teaching CCU rates ${ }^{[5]}$. This is despite implementation of practice standards to reduce the number of infections, such as the use of silver-alloy catheters, education to prevent tube kinking and looping, use of anchoring devices, maintaining the drainage bag below bladder level, and daily assessments for discontinuation of the devices ${ }^{[6]}$. Bacteria from the patient's perineum can gain access to the urinary bladder by ascending the external surface of the catheter ${ }^{[4]}$. Thus it is critical that appropriate catheter care be provided to these patients. Though staff in the CCU at the study hospital have provided catheter care, there may have been lapses in consistency of all recommended elements each shift, including consistent perineal/meatal care to prevent 
bacterial biofilm development on the extra-luminal surface of the catheter. There was an essential need to develop a process to ensure that consistent catheter care was provided each shift for patients who have urinary catheters.

\subsection{Literature review}

A review of the literature from 2008 through 2013 was conducted using CINAHL, MEDLINE, Academic Search Premier, and Cochrane databases to analyze studies related to urinary catheter care practices aimed at reducing CAUTIs. The majority of the studies were aimed at early discontinuation and reducing the overall number of catheters inserted, which have been well-known practices to reduce CAUTIs. Minimal studies addressed care practices to reduce infections once catheters were inserted. Of these studies, two were descriptive in design to determine risk factors for CAUTIs during daily care routines ${ }^{[7,8]}$; two focused on education interventions to teach clinicians the care techniques to prevent CAUTIs ${ }^{[9,10]}$; two were non-random control trials to address a bundled approach somewhat similar to this present study ${ }^{[11,12]}$; and one of the above studies also focused on the use of checklists similar to this present study ${ }^{[12]}$. Only three studies that were found were conducted in the United States.

Fink et al. ${ }^{[7]}$ and Tsuchida et al. ${ }^{[8]}$ conducted descriptive studies to identify common risk factors for CAUTIs. Fink et al. used a self-reporting survey to determine each hospital's degree of adherence to national CAUTI prevention standards, while Tsuchida et al. conducted a prospective observational study to identify patterns of care that may lead to CAUTI risk. In the first study, Fink et al. surveyed 255 hospitals in the United States and Canada with 75 responses returned. Results demonstrated much variability among the hospitals in practices of meatal care and anchoring, supporting the need for improvement in standardization of catheter care to prevent CAUTIs ${ }^{[7]}$. In Tsuchida et al.'s study, 555 adult patients with urinary catheters from five hospitals in Japan were observed to determine patterns of care provided to these patients to identify at-risk behaviors. Those hospitals with the highest CAUTI rates were also the ones with the lowest percentage of compliance in following standard CAUTI prevention practices ${ }^{[8]}$.

Altun and Karakoc ${ }^{[9]}$ and Oman et al. ${ }^{[10]}$ conducted educational interventions to reinforce catheter care practices to study the impact this had on CAUTI rates. Altun and Karakoc conducted an interactive workshop with a small sample of nurses, whereas Oman et al. conducted an education intervention with a mandatory web-based program for an entire hospital clinical staff, and a focused classroom education session for a select group of nurses. In both studies there was no correlation between provision of an educational intervention to teach catheter care, and a decrease in CAUTI rates ${ }^{[9,10]}$. Though education is an important component, this intervention alone may not have a direct impact on reducing CAUTI rates.

An intervention during the actual catheter care delivery may have more potential in decreasing CAUTI rates than education alone, such as the bundled approach conducted by Leblebicioglu, et al. ${ }^{[11]}$ and Mara, et al. ${ }^{[12]}$ Leblebicioglu et al. conducted a non-randomized control study from September 2003 to June 2011 in 13 intensive care units (ICUs) in 10 hospitals in Turkey ${ }^{[11]}$. In each of the ICUs, active surveillance data was obtained for three months prior to the intervention to collect baseline CAUTI incidence rates. Staffs were then provided with catheter care bundling education to include appropriate criteria for aseptic insertion of catheters, anchoring the catheter, maintaining a closed system, preventing kinks in the tubing, empting the drainage bag frequently, and meatal cleansing as part of routine hygiene. The catheter care bundle was implemented by staff during the intervention period. Daily observations of catheter care were conducted to monitor compliance with the care bundle. Findings supported the use of a care bundle to decrease CAUTIs ${ }^{[1]}$.

Mara et al. ${ }^{[12]}$ used a different bundling approach to reduce CAUTIs. This was a non-randomized control study conducted from January 2008 to July 2010 in a hospital in Sao Paulo, Brazil with a 38-bed ICU and two 20-bed step down units. A bundled approach with a checklist was used for the insertion process to include use of an insertion cart, hand hygiene, chlorhexidine meatal washing, sterile gloves and field, and only one attempt at insertion per catheter used. Additionally, one nurse from each of the units rounded daily on the catheter patients and discussed possible discontinuation of the 
catheters with the physicians as part of the bundle. Similar to Leblebicioglu et al. ${ }^{[1]}$, findings of this study supported the use of a bundled approach to reduce CAUTIs ${ }^{[12]}$.

Gaps exist in the literature that study the use of bundling practices to reduce CAUTIs and to improve care practices provided to patients with urinary catheters. The majority of the studies in the CAUTI literature are related to the wellestablished practices of minimizing the number of insertions and early discontinuation. There is minimal information in the literature related to the care of catheters while they reside in patients and prevention of CAUTIs ${ }^{[7-12]}$.

\subsection{Purpose of study}

The purpose of this quasi-experimental study was to test the effect of using a bundled approach of catheter care practices for a 3-month period to reduce the occurrence of CAUTIs in adult CCU patients (18 years of age or greater) who had indwelling urinary catheters. A catheter had to have been in place for at least two calendar days after patient admission to the CCU in order for a CAUTI to be attributed to the care received by the CCU staff ${ }^{[3]}$. The research question investigated: What is the effect of performing a consistent prevention bundle on all patients in the adult CCU with urinary catheters to prevent CAUTIs as compared to patients who did not receive the prevention bundle in the prior year?

\subsection{Definition of terms}

The conceptual definition of a "bundle" is to perform all elements of care at one time along with completion of a checklist to ensure that each element is included in that care ${ }^{[13]}$. For purposes of this study, the operational definition of a prevention bundle is to perform the following elements of catheter care each shift in the critical care unit with the use of a bedside checklist to mark each element as care is rendered: (a) Ensure drainage bag is below the level of the bladder, (b) ensure drainage bag is not touching the floor, (c) ensure tubing is free of kinks and/or dependent loops, (d) assess for presence of catheter anchor and apply one if absent, (e) provide perineal/meatal care and catheter tube cleansing each shift with disposable cleansing wipes, and (f) discuss possibility of catheter discontinuation with the physician. The definition of a CAUTI is: “An indwelling catheter in place for $>2$ calendar days, with day of device placement being Day 1, and catheter is in place on the date of event and at least 1 of the following signs or symptoms: fever $\left(>38^{\circ} \mathrm{C}\right)$; suprapubic tenderness, costovertebral angle pain or tenderness and a positive urine culture of $>105$ colony-forming units (CFU)/ml with no more than two species of microorganisms" ${ }^{[3]}$.

The CAUTI's at the study hospital are confirmed by the hospital laboratory and validated by members of the infection control department after record audit.

\subsection{Theoretical framework}

The Institute for Healthcare Improvement Framework for Spread was chosen for the conceptual framework for this study ${ }^{[14]}$. The Framework for Spread was created with the purpose of developing and implementing a method to spread positive changes within and outside a healthcare organization ${ }^{[14]}$. The Framework for Spread is based on Rogers's work Diffusion of Innovations ${ }^{[15]}$, which proposes that innovations may take lengthy amounts of time to be communicated, spread, and adopted by others; and this spread should be accelerated so that others can benefit from the innovations sooner.

There are several main points that must exist for the Framework of Spread to be successful, including recognition by leadership to support improvement, an aim for spread, a target population, a time frame for completion, a communication plan, early adopters to encourage others, a method to measure improvement, a method to provide feedback and a method to carry results forward to other areas ${ }^{[14]}$. Nursing leadership at the study hospital has recognized the need for and championed the development of a study to improve CAUTI rates in the CCU. Nurses in the CCU will be chosen as the early adopters for implementation of the prevention bundle intervention. The CAUTI measure currently collected and reported to CMS for critical care will be a mandated measure to be collected and reported for all inpatient acute care units as of January $2015^{[3]}$. It will be essential to follow this framework to test an intervention within the CCU; and if successful, 
quickly spread as a change throughout all acute care units to minimize further hospital acquired CAUTIs. The CCU nurses will be instrumental in assisting the nurse leaders to educate and to spread the change to their peers on other inpatient units. Monthly CAUTI incidence will be used to evaluate improvement.

\subsection{Study design}

A quasi-experimental design was employed to compare pre-intervention CAUTI incidence rates with post-intervention CAUTI rates. The intervention consisted of a prevention bundle and bedside checklist. Due to the inability to withhold catheter care from a control group, a retrospective record review was used to compare the pre and post data.

Table 1. Critical care patient characteristics

\begin{tabular}{|c|c|c|c|}
\hline \multicolumn{2}{|c|}{ Pre-Intervention Group } & \multicolumn{2}{|c|}{ Intervention Group } \\
\hline Age $(n=317)$ & & Age $(n=310)$ & \\
\hline$<65=137(43.2 \%)$ & & $<65=143(46.1 \%)$ & \\
\hline $65-74=76(24.0 \%)$ & & $65-74=58(18.7 \%)$ & \\
\hline $75-84=69(21.8 \%)$ & & $75-84=72(23.2 \%)$ & \\
\hline $85+=35(11.0 \%)$ & & $85+=37(11.9 \%)$ & \\
\hline Gender & & Gender & \\
\hline $\mathrm{M}=190(59.9 \%)$ & & $M=188(60.6 \%)$ & \\
\hline $\mathrm{F}=127(40.1 \%)$ & & $\mathrm{F}=122(39.4 \%)$ & \\
\hline Foley Days/Patient Days & & Foley Days/Patient Days & \\
\hline $976 / 1088$ & & $931 / 1189$ & \\
\hline Number of CAUTIs & & Number of CAUTIs & \\
\hline 8 & & 4 & \\
\hline Diagnosis Groupings (n) & & Diagnosis Groupings (n) & \\
\hline Cardiac disease & 11 & Cardiac disease & 12 \\
\hline Cardiothoracic procedure & 106 & Cardiothoracic procedure & 115 \\
\hline Digestive/liver system & 8 & Digestive/liver system & 9 \\
\hline Drug/alcohol dependency & 11 & Drug/alcohol dependency & 19 \\
\hline $\begin{array}{l}\text { Esophageal/Stomach/Bowel } \\
\text { procedure }\end{array}$ & 11 & $\begin{array}{l}\text { Esophageal/Stomach/Bowel } \\
\text { procedure }\end{array}$ & 4 \\
\hline Infectious disease & 5 & Infectious disease & 6 \\
\hline Kidney \& urologic & 7 & Kidney \& urologic & 6 \\
\hline Neurologic disease & 18 & Neurologic disease & 21 \\
\hline Neurologic procedure & 18 & Neurologic procedure & 22 \\
\hline Respiratory disease & 29 & Respiratory disease & 24 \\
\hline Septicemia & 21 & Septicemia & 24 \\
\hline Trauma & 19 & Trauma & 14 \\
\hline Vascular disease & 8 & Vascular disease & 5 \\
\hline Vascular procedure & 6 & Vascular procedure & 7 \\
\hline Other disease & 18 & Other disease & 11 \\
\hline Other procedure & 21 & Other procedure & 11 \\
\hline Total & 317 & Total & 310 \\
\hline
\end{tabular}

\subsubsection{Setting and sample}

The setting for this study was a 20-bed CCU at an acute care hospital in the Northeast where all adult patients (18 years of age or greater) requiring a critical level of care were admitted, including all medical, surgical, trauma and cardio-thoracic patients. There were two sample groups for this study: a pre-intervention comparison group and an intervention group. The 
comparison group consisted of all patients with urinary catheters admitted to the adult CCU from October 1, 2012 through December 31, 2012 (First quarter fiscal year 2013). The intervention group consisted of all patients with urinary catheters admitted to the adult CCU from October 1, 2013 through December 31, 2013 (First quarter fiscal year 2014). The first quarters of both years were used for equivalent comparison groups because the critical care population characteristics at the study hospital are cyclical from the perspective of patient volumes and diagnoses. Selection bias was eliminated by including all patients with indwelling catheters in the study who did not have a UTI when entering the study. Exclusion criteria for this study included patients admitted to the CCU who were less than 18 years of age, those who did not have indwelling catheters, or those who had been admitted with a UTI or developed a UTI prior to the two calendar day criteria ${ }^{[3]}$. Additionally, any patients with suprapubic catheters or intermittent catheterizations were excluded. See Table 1 for CCU patient characteristics for pre-intervention and intervention periods.

Sample size was based on number of catheter days for all patients combined and the corresponding CAUTI incidence rate. Catheter days were determined by counting the number of patients who had urinary catheters in place each day of the week at the same time of day; and then each day's numbers were added for a monthly total ${ }^{[5]}$. This sampling method was used to account for variation in population sizes each month in the CCU when comparing the number of CAUTIs over time ${ }^{[16]}$. The outcome variable measured was CAUTI incidence rate. The NHSN ${ }^{[3]}$ formula to calculate the incidence rate for CAUTIs was used. This formula is the number of new CAUTIs divided by the total number of catheter days multiplied by 1000 for each of the pre- and post study time periods (CAUTIs/Cath Days $\times 1000=$ Rate).

\subsubsection{Ethical considerations}

This study was approved by the study hospital Institutional Review Board (IRB). Neither patient names nor identification numbers were collected thus assuring patient confidentiality and anonymity. Catheter days and confirmed numbers of CAUTIs were collected by one of the hospital infection control coordinators, and only the numbers, without identifying data, were given to the investigator.

\section{Method}

\subsection{Process}

Prior to initiation of the study, all CCU nurses and aides attended an educational session as part of the Framework of Spread plan to inform them of the study goals and the care process. Training was conducted in a classroom setting by the nurse manager and infection control coordinator, both of whom took part in the literature review of best practice standards. The teach-back method was used to ensure staff understood the education and care process. Content included the current CAUTI incidence rate, research evidence, the elements of the care bundle and required frequency of performing the bundle, use of the bedside checklist to validate care provided, and use of three disposable cleansing wipes to cleanse the perineal/meatal area and catheter tubing. The bedside checklist contained check boxes for each element of the bundle to mirror care practices, and the nursing staff used the checklist each shift as a reminder to provide the entire bundle. The care bundle elements included: (a) ensuring that the drainage bag was below the level of the bladder; (b) ensuring that the drainage bag was not touching the floor; (c) ensuring that the tubing was free of kinks and/or dependent loops; (d) assessing for the presence of a catheter anchor; (e) performing perineal/meatal care once a shift using the three disposable wipes; and (f) discussing with the physician the possibility of discontinuing the catheter. Staff was fully engaged in the need to improve overall performance in caring for patients with indwelling catheters and to adopt the above methods for CAUTI prevention.

To ensure that care was provided each day and to control for variability in perineal/meatal care, two clear plastic bags were filled with three of the disposable wipes each, and the bags were placed in the same spot in each room where the bags were easily visualized. Dedicated staff was in-serviced on how to appropriately prepare these bags of wipes and where to place the bags in the patient rooms. The charge nurse audited each catheter patient room an hour prior to the end of shift to note 
if care had been given. The charge nurse audit mirrored the bedside checklist. If care had not been given, the charge nurse reminded the responsible nurse to provide the care. The nurse manager and assistant nurse manager also conducted weekly audits of catheter care provided by the staff to ensure that care was provided correctly.

\subsection{Data collection}

The catheter day number was taken from a patient census log that was maintained in the CCU by the unit clerk at the beginning of the day shift. This unit clerk recorded the number of patients each day that had urinary catheters in place. An infection control coordinator collected the catheter day information daily. Urine cultures were ordered only if a patient was symptomatic for a CAUTI. The hospital laboratory reported all positive urine cultures and urinalyses to the infection control department. Two infection control coordinators then independently reviewed patient records for evidence of CAUTIs. Once the independent reviews were completed, the director of the infection control department conferred with the infection control coordinators and each infection was reviewed against the NHSN ${ }^{[3]}$ surveillance definition. Group consensus was then achieved to determine presence of a CAUTI. The infection control director attended formal education for the NHSN surveillance definition of CAUTIs offered by the Association for Professionals in Infection Control and Epidemiology, the Society for Healthcare Epidemiology of America, and the Centers for Disease Control and Prevention.

\section{Results}

The Wilcoxin signed rank test was used to compare CAUTI rates between the pre- and post- intervention time periods. An $\alpha$-level of $p<.05$ was used. CAUTI incidence rates for the two time periods are displayed in Table 2 below. Results were not statistically significant, with $p=.285$. However, results were clinically significant. Overall, there was a $50 \%$ reduction in CAUTI incidence when comparing the pre-intervention period with the study period results.

Table 2. CAUTI incidence rates during pre-intervention and intervention time periods

\begin{tabular}{lll}
\hline Month & Pre-Intervention Period 2012 & Intervention Period 2013 \\
\hline Oct & 8.3 & 3.4 \\
Nov & 13.3 & 3.2 \\
Dec & 3.2 & 6.2 \\
Overall & 8.2 & 4.3 \\
\hline
\end{tabular}

Note. Incidence rates $=$ number of CAUTs $\div$ number of catheter days $\times 1000$

\section{Discussion}

Given the seriousness of CAUTIs in the critically ill population, decreasing the CAUTI rate by $50 \%$ was a tremendous leap forward in the study hospital's efforts to minimize this nosocomial infection. This study also provided standardization in nursing practice when caring for patients with urinary catheters. The bedside checklist provided further consistency in care as a reminder to staff to perform all aspects of CAUTI prevention.

Limitations in this study included lack of randomization for group assignments. For ethical reasons, standard catheter care could not be withheld from CCU patients, thus eliminating the possibility of a true control group for this study. Further, it would not be feasible from a nursing work-flow perspective to effectively sort CCU patient assignments based on bundled versus non-bundled catheter care to form a control group. Finally, results were collected on adult patients in CCU and as such, results are not generalizable to pediatric patients.

A possible confounding variable in this study was the use of chlorhexidine wipes. During the pre-intervention time period, patients with central lines were cleansed daily with chlorhexidine wipes, which comprised only $75 \%$ of the CCU patients who also had indwelling urinary catheters. As such, the other $25 \%$ of patients in the pre-intervention control group with 
urinary catheters during this time period were not cleansed with chlorhexidine wipes. During the study/intervention period of October 2013 through December 2013, this process was changed to all patients cleansed with chlorhexidine wipes. This included $100 \%$ of patients with urinary catheters. This may have also had a positive impact in reducing CAUTIs, although the chlorhexidine wipes were never used on mucosal areas such as the perineum $/$ meatus.

\subsection{I mplications for nursing}

Catheter care is completed primarily by nursing staff, but minimal studies exist in the literature that support specific nursing care to prevent the occurrence of CAUTIs for patients with indwelling catheters. This prevention bundle research has aided in filling that gap. The bundled care approach supported a clinically significant reduction in CAUTI incidence by $50 \%$. Using the Framework of Spread ${ }^{[14]}$, results of this study will be shared with other patient care units in the study hospital to spread the CAUTI prevention bundle and optimize care provided to patients with indwelling urinary catheters. The CCU nursing staff will be utilized to spread and champion the use of the prevention bundle with their peers on the general medical /surgical units.

\subsection{Recommendations}

Additional research should be conducted to test the efficacy of the use of the CAUTI prevention bundle on other patient care units in other health care settings.

\section{Competing interests}

The authors declare that they have no competing interests.

\section{References}

[1] Joint Commission Resources. Clinical improvement strategies: Preventing catheter-associated urinary tract infections. Oakbrook Terrace, Illinois: Joint Commission Resources, Inc. 2011.

[2] Lo, E., Nicolle, L., Classen, D., Arias, K., Podgorny, K., Anderson, D. J., et al. Strategies to prevent catheter-associated urinary tract infections in acute hospitals. [Supplement]. Infection Control and Hospital Epidemiology. 2008; 29: S41-S50. PMid: 18840088. http://dx.doi.org/10.1086/591066

[3] National Health Safety Network. (2013a). July 2013 CDC/NHSN protocol clarifications. Available from: http://www.cdc.gov/nhsn/PDFs/pscManual/7pscCAUTIcurrent.pdf

[4] Shuman, E.K., Chenoweth, C. E. Recognition and prevention of healthcare-associated urinary tract infections in the intensive care unit. [Supplement]. Critical Care Medicine. 2010; 8(8): S373-S379. PMid: 20647795. http://dx.doi.org/10.1097/CCM.0b013e3181e6ce8f

[5] National Health Safety Network. (2013b). National Healthcare Safety Network report, data summary for 2011, device-associated module. Available from: http://www.cdc.gov/nhsn/PDFs/dataStat/NHSN-Report-2011-Data-Summary.pdf

[6] Gould, C. V., Umscheid, C. A., Rajender, K., Agarwal, R. K., Kuntz, G. Pegues, D. A., The Healthcare Infection Control Practices Advisory Commmittee (HICPAC). Guidelines for prevention of catheter-associated urinary tract infections, 2009. Available from: http://www.cdc.gov/hicpac/cauti/001_cauti.html

[7] Fink, R., Gilmartin, H., Richard, A., Capezuti, E., Boltz, M., Wald, H. Indwelling urinary catheter management and catheter associated urinary tract infection prevention practices in Nurses Improving Care for Healthsystem Elders hospitals. American Journal of Infection Control. 2012; 40(8): 715-720. http://dx.doi.org/10.1016/j.ajic.2011.09.017

[8] Tsuchida, T., Makimoto, K., Ohsako, S., Fujino, M., Kaneda, M., Miyazaki, T., et al. Relationship between catheter care and catheter associated urinary tract infections at Japanese general hospitals: A prospective observational study. International Journal of Nursing Studies. 2008; 45(3): 352-361. http://dx.doi.org/10.1016/j.ijnurstu.2006.10.006

[9] Altun, I., Karakoc, A. The impact of an interactive workshop on the management of urinary catheterization on nurses. Journal of Urologic Nursing. 2010; 4(3): 125-132. http://dx.doi.org/10.1111/j.1749-771X.2010.01107.x

[10] Oman, K.S, Makic, M. B. F., Fink, R., Schraeder, N., Hulett, T., Keech, T., et al. Nurse-directed interventions to reduce catheter-associated urinary tract infections. American Journal of Infection Control. 2012; 40(6): 548-553. http://dx.doi.org/10.1016/j.ajic.2011.07.018 
[11] Leblebicioglu, H., Gulden, E., Rosenthal, V.D., Nevzat-Yalcin, A., Akan, O.A., Sirmatel, F., et al. Impact of a multidimensional infection control approach on catheter associated urinary tract infection rates in adult intensive care units in 10 cities in Turkey: International Nosocomial Infection Control Consortium (NICC) findings. American Journal of Infection Control. 2013; 41(10): 885-891. http://dx.doi.org/10.1016/j.ajic.2013.01.028

[12] Mara, A. R., Camargo, T.Z., Goncalves, P., Sogayar, A.M., Moura, D. F., Guastelli, L. R., et al. Preventing catheter-associated urinary tract infections in the zero-tolerance era. American Journal of Infection Control. 2011; 39(10): 817-822. http://dx.doi.org/10.1016/a.ajic.2011.01.013

[13] Institute for Healthcare Improvement. How-to Guide: Prevent catheter-associated urinary tract infections. Cambridge, MA: Institute for Healthcare Improvement. 2011

[14] Massoud, M. R, Nielsen, G. A., Nolan, K., Nolan, T. Schall, M. W., Sevin, C. A framework for spread: From local improvements to system-wide change. IHI innovation series white paper. Cambridge, MA: Institute for Healthcare Improvement. 2006.

[15] Rogers, E. M. Diffusion of innovations, 4th ed. [DX Reader version]. Available from: http://www.appple.com/apps/ibook. 1995.

[16] Centers for Disease Control and Prevention. Principles of epidemiology in publichealth practice. 2012. Available from the Centers for Disease Control and Prevention website: http://www.cdc.gov/osels/scientific_edu/ss1978/lesson3/section2.html 